\title{
Development of Negative Electrode for Calcium-Ion Battery Using Marimo Nano Carbon
}

\author{
Y. Nishimura ${ }^{\mathrm{a}}$, K. Nakagawa ${ }^{\mathrm{a}, \mathrm{b}}$ \\ a Department of Science and Engineering, Kansai University, 3-3-35, Yamate-cho, Suita, \\ Osaka, 564-8680, Japan \\ ${ }^{\mathrm{b}}$ Department of Science and Engineering and High Technology Research Core (HRC), \\ Kansai University, 3-3-35, Yamate-cho, Suita, Osaka, 564-8680, Japan
}

The problem of calcium-ion batteries (CIBs) using graphite electrodes is that the charge/discharge capacity cannot be sufficiently obtained due to the poor reactivity of $\mathrm{Ca}^{2+}$. In this study, we investigated Marimo Nano Carbon (MNC) as a new negative electrode material for CIBs. MNC was an aggregate of carbon nanofilaments (CNFs) and it was synthesized by decomposition of ethylene using oxidized diamond-supported Pd catalyst. When the charge and discharge capacity using MNC as negative electrode was larger than that of the natural graphite. In addition, the intercalation of $\mathrm{Ca}^{2+}$ between the graphene layers was confirmed by XRD measurement.

\section{Introduction}

Calcium-ion batteries (CIBs) use $\mathrm{Ca}^{2+}$ for charge carriers instead of $\mathrm{Li}^{+}$and have great potential as alternatives to lithium-ion batteries (LIBs) (1-3). Since it uses divalent cations, it has the potential to obtain twice the capacity of LIBs. In addition, compared with $\mathrm{Mg}^{2+}$, the standard electrode potential of $\mathrm{Ca}$ is approximately $0.5 \mathrm{~V}$ lower $(-2.87,-2.37 \mathrm{~V}$ vs. SHE for $\mathrm{Ca}, \mathrm{Mg}$ ), and it can use a high operating voltage.

However, there are some problems in the development of CIBs. Since divalent cations such as $\mathrm{Ca}^{2+}$ have a stronger Lewis acidity than $\mathrm{Li}^{+}$, they form a strong solvation structure with solvent molecules in the electrolyte, and also have large desolvation energy $(4,5)$. Therefore, $\mathrm{Ca}^{2+}$ are less reactive than $\mathrm{Li}^{+}$.

For this reason, we used Marimo Nano Carbon (MNC) as a new negative electrode material. MNC is an aggregate of carbon nanofilaments (CNFs) (6). This CNFs have coinstacked structure in which graphene layers are stacked similar to graphite and the edge plane is exposed. Generally, graphite used for LIBs has a spheroidizing treatment on the edge plane in order to suppress excessive decomposition of the electrolytes, and as a result, reactivity is suppressed (7). Hence, we expected that MNC negative electrode would more intercalate $\mathrm{Ca}^{2+}$ between the graphene layers of CNFs and indicated larger charge/discharge capacity than that of the graphite negative electrode.

In this paper, we synthesized CNFs oxidized diamond-supported catalyst on decomposition of ethylene by chemical vapor deposition (CVD) method, and We evaluated the performance of MNC electrodes as negative electrode materials for CIBs.

\section{Experimental}




\section{Preparation of catalyst for synthesis CNFs}

Oxidized diamond (Diamond Innovations Co., Ltd.) was used as the catalyst supports. Oxidized diamond has oxygen-containing functional groups arranged regularly, so that nano metal particles can a highly dispersed on it (8). Therefore, oxidized diamondsupported Pd catalyst (Pd loading: $5 \mathrm{wt} \%$ ) for CNFs synthesis was prepared by the metal nano-colloidal method (9). Diamond oxide was added to deionized water and stirred for 30 min under ultrasonic treatment. $\mathrm{PdCl}_{2}$ as a precursor and citric acid as a dispersing agent were added, and this solution was stirred for 30 min under ultrasonic treatment. $\mathrm{NaBH}_{4}$ as a reducing agent was added to the solution. After stirring for 30 min under ultrasonic treatment, the supernatant was removed from the solution by centrifugation and dried at $333 \mathrm{~K}$. Supported catalysts were calcined at $723 \mathrm{~K}$ for $5 \mathrm{~h}$ in $\mathrm{N}_{2}$ prior to the reaction.

CNFs were synthesized by the CVD method using a horizontal rotating fluidized bed reactor (10). Oxidized diamond-supported Pd catalyst was used $200 \mathrm{mg}$ and uniformly distributed in a quartz boat. After inserting quartz boat into the center of the quartz tube, the reactor was elevated temperature at $873 \mathrm{~K}$, under $\mathrm{N}_{2}$ atmosphere. Afterwards, $30 \mathrm{~mL} /$ $\min \mathrm{C}_{2} \mathrm{H}_{4}$ was introduced at $873 \mathrm{~K}$. The reaction time was $1 \mathrm{~h}$.

\section{Characterization of MNC}

Surface structures of MNC were observed using field emission scanning electron microscopy (FE-SEM; Hitachi, Ltd. S-4800), and internal structures of synthesized CNFs were observed using transmission electron microscopy (TEM; JEOL Ltd. JEM-2010K). The degree of graphitization of synthesized CNFs were measured by Raman spectroscopy (Raman; JASCO, NRS-3000). The rate of surface functional group of CNFs was measured by X-ray photoelectron spectroscopy (XPS; JEOL Ltd. PS-9000MX). The interlayer spacing $\left(\mathrm{d}_{002}\right)$ of synthesized CNFs were measured by X-ray diffraction (XRD; Rigaku, SmartLab). The specific surface area and the ratio of the mesopore volume to the total volume of synthesized CNFs were measured by the nitrogen adsorption method at $77 \mathrm{~K}$ (BELSORP-mini; Nippon Bel, Japan).

\section{Electrochemical measurements}

MNC were used as a working electrode and an activated carbon fiber cloth (Kynol; Gunei Chemical Industry, Ltd.) was used as a counter electrode. Spherical natural graphite (CGB-10; Nippon Graphite Industries, Ltd.) was used as comparative material. Cellulose fiber (ADVANTEC No.5, Toyo, Ltd.) was used as a separator. The electrolytes were prepared by dissolving a $\mathrm{Ca}\left(\mathrm{ClO}_{4}\right)_{2}$ at $1 \mathrm{M}$ concentration in ethylene carbonate (EC): diethyl carbonate $(\mathrm{DEC})=1: 1(\mathrm{vol} / \mathrm{vol})$, and $\gamma$-butyrolactone (GBL). These were purchased from FUJIFILM Wako Pure Chemical Ltd.

In order to calculate charge/discharge capacity of each electrolyte, charge and discharge measurement was performed (HZ-7000, Hokuto Denko). The measurement was conducted out at a scanning potential of 0 to $3.5 \mathrm{~V}$ and performed 3 cycles. In addition, intercalation of $\mathrm{Ca}^{2+}$ into graphene layers of $\mathrm{MNC}$ anode was measured by XRD.

\section{Results and Discussions}




\section{Characterization of MNC}

Fig. 1 shows SEM images of synthesized CNFs over oxidized diamond-supported $\mathrm{Pd}$ catalyst. From these images, it can be seen that CNFs are densely generated and there are many pores between the fibers. The diameters of CNFs were approximately in the range of 5-100 nm.
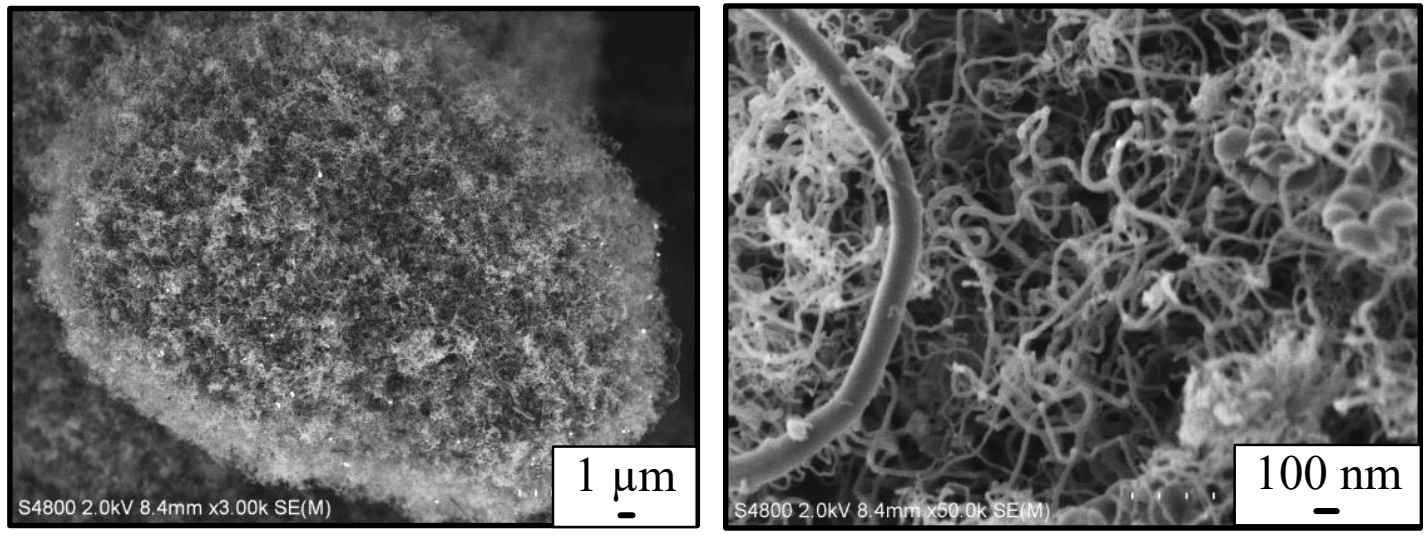

Figure 1. SEM images of synthesized CNFs.

Fig. 2 (a) shows TEM image of synthesized CNF over oxidized diamond-supported Pd catalyst and model of coin-stacked structure CNF. It seems that Synthesized CNFs had coin-stacked structures which is stacked graphene wall perpendicularly to the nanofilaments axis. Fig 2 (b) shows model diagram of coin-stacked CNF.

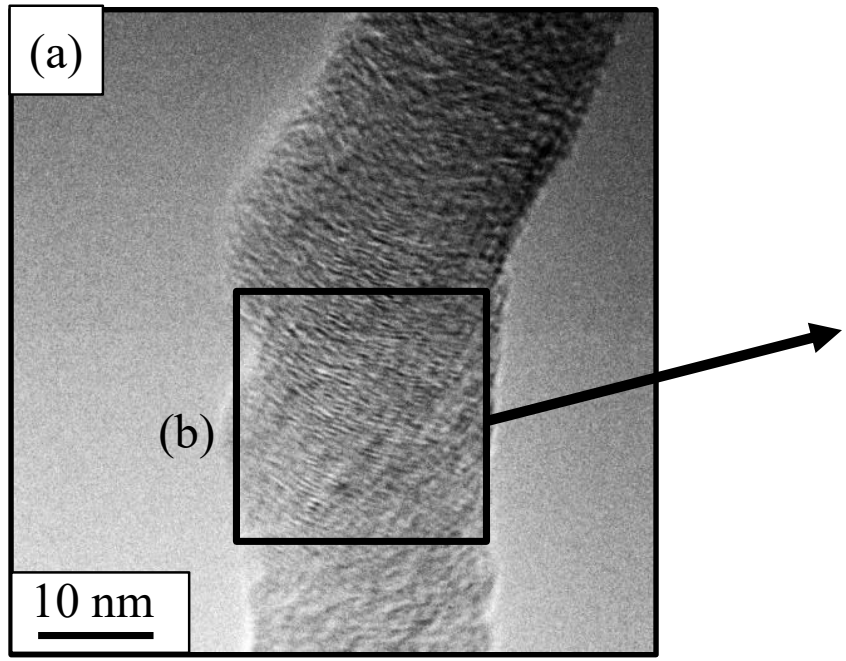

\section{(b)}

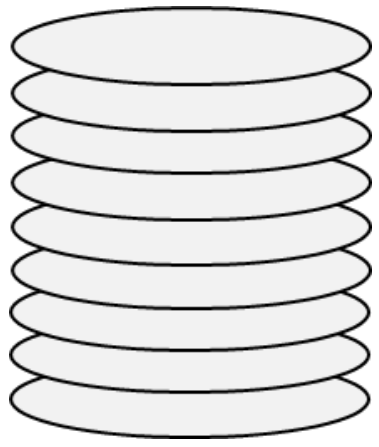

Figure 2. (a) TEM image of synthesized CNF and (b) model of coin-stacked structure CNF.

TABLE I. Characteristic data of MNC and Spherical natural graphite.

\begin{tabular}{cccccc}
\hline Samples & $\mathbf{I}_{\mathbf{G}} / \mathbf{I}_{\mathbf{D}}[-]$ & $\mathbf{C}-\mathbf{C}[\%]$ & $\mathbf{d}_{\mathbf{0 0 2}}[\AA]$ & $\mathbf{S}_{\text {BET }}\left[\mathbf{m}^{2} / \mathbf{g}\right]$ & $\mathbf{V}_{\text {meso }}\left[\mathbf{c m}^{2} / \mathbf{g}\right]$ \\
\hline $\begin{array}{c}\text { MNC } \\
\begin{array}{c}\text { Spherical natural } \\
\text { graphite }\end{array}\end{array}$ & 1.14 & 78.1 & 3.45 & 188 & 0.287 \\
\hline
\end{tabular}


TABLE I shows Characteristic data of MNC and Spherical natural graphite. First, $\mathrm{I}_{\mathrm{G}} / \mathrm{I}_{\mathrm{D}}$ was calculated by Raman spectroscopy. The peaks at $1580 \mathrm{~cm}^{-1}$ and $1350 \mathrm{~cm}^{-1}$ correspond to the $\mathrm{G}$ (graphite)-band and the $\mathrm{D}$ (disorder)-band, respectively, and the $\mathrm{I}_{\mathrm{G}} / \mathrm{I}_{\mathrm{D}}$ is represented by the ratio of the intensity of the G-band to the D-band (11). The values of $\mathrm{I}_{\mathrm{G}} / \mathrm{I}_{\mathrm{D}}$ for MNC and Spherical natural graphite were 1.14 and 3.36, respectively. This result is because CNFs are synthesized at a relatively low temperature, so that the crystal structure is not sufficiently developed and the edge plane has defects such as oxygen-containing functional groups.

Second, the surface functional group ratio of the synthesized CNFs and Spherical natural graphite were measured by XPS. The values in the TABLE I indicate the ratio of $\mathrm{C}-\mathrm{C}$ bonds $\left(\mathrm{sp}^{2}, 284.4 \mathrm{eV}\right)$ in the $\mathrm{C} 1 \mathrm{~s}$ spectrum. The rate of C-C bond of CNF and Spherical natural graphite was $78.1 \%$ and $81.0 \%$, respectively. This result coincided with the result of Raman measurement. From the result of Raman and XPS, it was able to confirm that there was a correlation between the degree of graphitization and the rate of surface functional group. Therefore, CNFs are considered to have more exposed edge plane than that of spherical natural graphite.

Third, d-spacing $\left(\mathrm{d}_{002}\right)$ of synthesized CNFs and Spherical natural graphite were measured by XRD. From the results of XRD, the values of $\mathrm{d}_{002}$ of each sample were 3.45 $\AA$ and $3.35 \AA$, respectively. Therefore, synthesized CNFs had similar d-spacing of graphite.

Finally, the specific surface area $\left(\mathrm{S}_{\mathrm{BET}}\right)$ and the ratio of the mesopore volume to the total volume $\left(\mathrm{V}_{\text {meso }}\right)$ of synthesized CNFs and Spherical natural graphite were measured by the nitrogen adsorption method at $77 \mathrm{~K}$. The synthesized CNFs had a larger specific surface area and the mesopore volume than that of Spherical natural graphite.

\section{Electrochemical measurement}

TABLE II . Charge/discharge capacity of MNC and Spherical natural graphite electrodes in each electrolyte.

\begin{tabular}{c|cc|cc}
\hline Solvent & \multicolumn{2}{|c|}{ EC-DEC } & \multicolumn{2}{c}{ GBL } \\
\hline Samples & $\begin{array}{c}\text { Charge } \\
{[\mathbf{m A h} / \mathbf{g}]}\end{array}$ & $\begin{array}{c}\text { Discharge } \\
{[\mathbf{m A h} / \mathbf{g}]}\end{array}$ & $\begin{array}{c}\text { Charge } \\
{[\mathbf{m A h} / \mathbf{g}]}\end{array}$ & $\begin{array}{c}\text { Discharge } \\
{[\mathbf{m A h} / \mathbf{g}]}\end{array}$ \\
\hline $\begin{array}{c}\text { MNC } \\
\begin{array}{c}\text { Spherical natural } \\
\text { graphite }\end{array}\end{array}$ & 6.3 & 4.9 & 98.9 & 20.2 \\
\hline
\end{tabular}

TABLE II shows the charge/discharge capacity of MNC and spherical natural graphite electrodes in each electrolyte. The order of charge/discharge capacity was MNC> Spherical natural graphite for each electrolyte. It is considered that this is because the MNC negative electrode with the highly active edge plane exposed reacted with $\mathrm{Ca}^{2+}$ in the electrolytes more than the Spherical natural graphite negative electrode. Further, the difference in charge/discharge capacity of each solvent may be due to the solution structure in each electrolyte.

Fig. 3 shows X-ray patterns of MNC negative electrode before and after charge in each organic electrolyte. In the GBL based electrolyte, the peak of (002) plane shifted after charging, confirming that the interlayer distance had expanded from $3.45 \AA$ to $4.29 \AA$. Recently, it has been reported that electrochemical intercalation of $\mathrm{Ca}^{2+}$ into graphite is possible $(3,12)$. Therefore, it was confirmed that $\mathrm{Ca}^{2+}$ intercalation proceeded between the graphene layers of CNFs after charging the MNC negative electrode. Furthermore, there 
was a correlation between the height of the peak after charging with each electrolyte and the charge/discharge capacity.

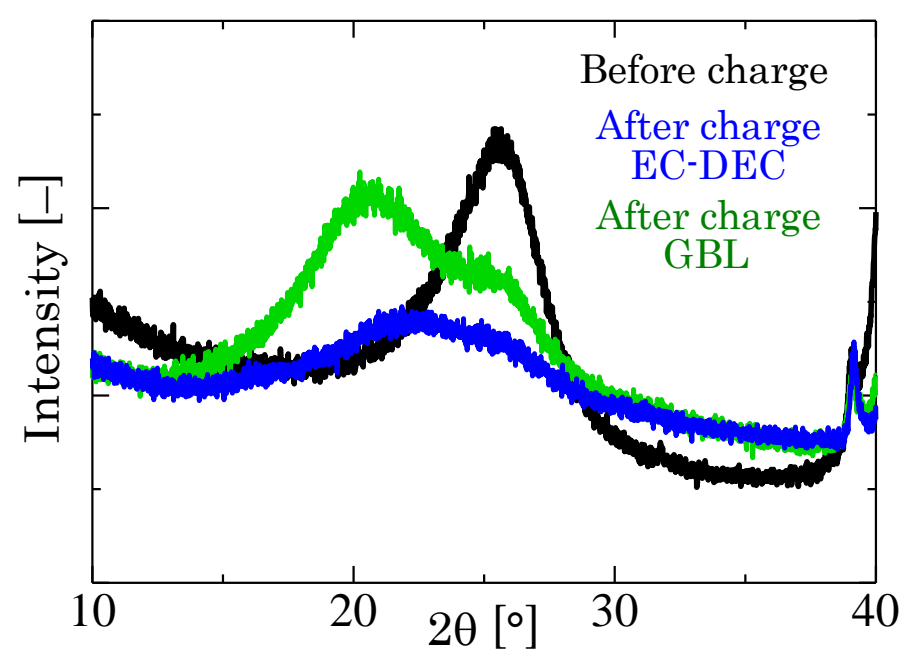

Figure 3. X-ray patterns of MNC negative electrode before and after charge in each electrolyte.

\section{Conclusions}

Synthesized CNFs had coin-stacked structures. Electrochemical measurements indicated that MNC negative electrode exhibits more charge/discharge capacity than that of graphite negative electrode. Result of XRD measurements, the peak of (002) plane of MNC negative electrode shifted after charging. Therefore, it was shown that $\mathrm{Ca}^{2+}$ can be intercalated between graphene layers of CNFs.

\section{Acknowledgments}

This work was supported by JSPS KAKENHI Grant Number 20K12274. In addition, the authors are grateful to Nippon Graphite Industries, Ltd., for supplying us with Spherical natural graphite for our research.

\section{References}

1. J. Muldoon, C. B. Bucur, and T. Gregory, Chem. Rev., 114, 23 (2014).

2. A. Ponrouch, J. Bitenc, R. Dominko, N. Lindahl, P. Johansson, and M. R. Palacin, Energy Stor. Mater., 20, 253 (2019).

3. S. J. Richard Prabakar, A. B. Ikhe, W. B. Park, K.-C. Chung, H. Park, K.-J. Kim, D. Ahn, J. S. Kwak, K.-S. Sohn, and M. Pyo, Adv. Sci., 6, 24 (2019).

4. J. Park, Z.-L. Xu, G, Yoon, S. K. Park, J. Wang, H. Hyun, H. Park, J. Lim, Y.-J. Ko, Y. S. Yun, and K. Kang, Adv. Mater., 32, 4 (2020).

5. J. D. Forero-Saboya, E. Marchante, R. B. Atraujo, D. Monti, P. Johansson, and A. Ponrouch, J. Phys. Chem., 123, 49 (2019).

6. Y. Kin, K. Nakagawa, H. Oda, and T. Ando, Trans. Mat. Res. Soc., 41, 1 (2016). 
7. K. Fukuda, T. Umeno, and Y. Hara, Materials Integration, 17, 1 (2004).

8. K. Nakagawa, T. Toriyama, G. Tsujino, T. Ando, H. Oda, ECS Trans., 50 (48), 55 (2013).

9. M. Eguchi, A. Okubo, S. Yamamoto, M. Kikuchi, K. Uno, Y. Kobayashi, M. Nishitani-Gamo, T. Ando, J. Power Sources, 195, 18 (2010).

10. G. Tsujino, H. Ota, M. N. Gamo, K. Nakagawa, H. Oda, TANSO, 2015, 266 (2015).

11. G. Katagiri, TANSO, 175, 304 (1996)

12. S. Wu, F. Zhang, and Y. B. Tang, Adv. Sci., 5, 8 (2018). 